\title{
The Effect of Xylitol on the Composition of the Oral Flora: A Pilot Study
}

\author{
Eva Söderling ${ }^{a}$ \\ Aino Hirvonen ${ }^{b}$ \\ Sara Karjalainen ${ }^{a}$ \\ Margherita Fontanac \\ Diana Catt ${ }^{\mathrm{d}}$ \\ Liisa Seppäe
}

\section{ABSTRACT}

Objectives: Our aim was to investigate the effect of short-term xylitol consumption on the microbial composition of plaque and saliva.

Methods: Twelve volunteers (22-38 yrs) harboring mutans streptococci (MS) participated in the randomized, double-blind, cross-over study. The experimental chewing gum contained $65 \%$ xylitol while the control gum contained $63 \%$ sorbitol and $2 \%$ maltitol w/w. The polyol dose was approximately $6 \mathrm{~g} /$ day. Stimulated saliva and plaque samples were collected before and after the two four-week test periods. The samples were cultured for MS, total streptococci, lactobacilli, and total facultatives. A part of the samples were subjected to DNA-DNA hybridizations of 14 microbial plaque species: Actinomyces naeslundii, A. viscosus, Fusobacterium nucleatum, Lactobacillus acidophilus, L. fermentum, L. paracasei, L. rhamnose, L. plantarum, Streptococcus gordonii, S. oralis, S. parasanguis, $S$. salivarius, S. sanguinis, Veillonella parvula.

Results: The MS counts of the plaque samples collected from "caries-prone" tooth sites decreased significantly $(P<.01)$ in the xylitol gum group but not in the sorbitol gum group. Also the plaque MS percentage decreased significantly in the xylitol gum group $(P<.01)$. The salivary MS counts did not decrease either in the xylitol or in the sorbitol gum groups. Nor were changes detected in the salivary levels of total streptococci or lactobacilli. The DNA-DNA hybridization assay revealed no study-induced changes in the microbial composition of the dental plaque.

Conclusions: Within the limitations of this pilot study, xylitol consumption reduced MS counts in plaque but appeared not to affect the microbial composition of plaque or saliva in general. (Eur $\mathrm{J}$ Dent 2011;5:24-31)

Key words: Xylitol; Mutans streptococci; Plaque; Oral flora; Chewing gum.

Adjunct Professor, Institute of Dentistry, University of Turku, Finland.

b DDS, Institute of Dentistry, University of Oulu, Finland. c Associate Professor, School of Dentistry, University of Michigan, USA.

d Postdoctoral Research Fellow, School of Dentistry, Indiana University, USA.

e Adjunct Professor, Institute of Dentistry, University of Oulu, Finland.
Corresponding author: Adjunct Professor Eva Söderling Institute of Dentistry, University of Turku, Lemminkäisenk. 2, FI-20520 Turku, Finland. Phone: +35823338351

Fax: +358 23338356

E-mail: eva.soderlingdutu.fi 


\section{INTRODUCTION}

Xylitol is a five-carbon polyol sweetener with specific, beneficial effects on oral health. Xylitol promotes mineralization by increasing the flow of saliva, which is a common effect of all polyol sweeteners. What is unique in xylitol is that it is practically nonfermentable by oral bacteria which counteracts low $\mathrm{pH}$-values in the oral cavity. ${ }^{1}$ The caries-preventive effect of xylitol in clinical studies could not be explained by the exclusion of fermentable sugars from the diet. ${ }^{2,3}$ In controlled clinical trials xylitol has performed better than sorbitol. 2,4,5 Recently, it was shown that xylitol syrup administration to children prevented early childhood caries, while no such effect was detected in the control group receiving high-sorbitol syrup. ${ }^{6}$ This study also demonstrated that not only chewing gum but also other xylitol vehicles can be effective in delivering xylitol.

The mechanism of action of xylitol on mutans streptococci (MS) is not fully known but habitual xylitol consumption, at high enough doses reduces counts of MS, apparently making plaque and mutans streptococci less adhesive to teeth. ${ }^{7}$ This appears to be reflected in a significant reduction in mother-child transmission of MS; in three of four published mother-child studies, habitual xylitol consumption by mothers resulted in reduced MS colonization in their children. ${ }^{8-11}$ According to in vitro studies MS can be regarded as target organisms of xylitol. ${ }^{12}$ Loesche et $\mathrm{al}^{13}$ showed that consumption of $5-7 \mathrm{~g}$ of xylitol in chewing gum reduced MS in both plaque and saliva but not counts of $S$. sanguis. Very little is actually known about the effects of xylitol on the oral flora apart from MS.

The hypothesis of the study was that in subjects showing xylitol-induced decreases in the counts of mutans streptococci no effects on the oral flora in general would be observed.

\section{MATERIALS AND METHODS}

Subjects

The clinical study was carried out in Oulu which is in Ostrobothnia, Finland. Twelve healthy dental and medical students, six males and six females with an age range of 22-38 years were recruited for the present study. Based on previous xylitol studies habitual xylitol consumption with daily $x y-$ litol doses $>5 \mathrm{~g}$ should reduce counts of mutans streptococci in all subjects. Thus twelve subjects were estimated to give enough power for statistical analyses. All subjects showed normal salivary flow rates of paraffin-stimulated saliva ( $>1 \mathrm{ml} / \mathrm{min}$ ) in all examinations. The subjects were screened for salivary MS (Dentocult SM Strip mutans test, Orion Diagnostica, Espoo, Finland), and all but three harbored high MS counts. All subjects had used xylitol chewing gum before the study started. Two of the subjects used xylitol gum approximately twice a week, the others on daily basis. In Finland, about half of the young adults have used xylitol chewing gums during most of their adolescence on a daily basis. Among university students this figure is even higher. The majority of the commercially available xylitol gums are pellet gums.

The Ethics Committee of the Northern Ostrobothnia Hospital District approved the study. Written consent was obtained from all subjects.

Experimental and control chewing gums

The experimental chewing gum (1.5 g/pellet) contained $65 \%$ xylitol $\mathrm{w} / \mathrm{w}$. The control gum was identical in pellet size but the main sweetener was sorbitol, with a concentration of $63 \%$. Maltitol ( $2 \%)$ was used in the control gum to make the texture of the gums similar. Both gums contained acesulfame $\mathrm{K}$ which was used to compensate for the differences in sweetness. The two gums had the same mild flavor and color. They were packed in identical plastic containers which were color-coded, blue and red. The codes of the test gums were kept by the gum manufacturer until the study was completed. The gum used during the washout periods was identical with the control gum but was packed in containers coded in green. The gums were manufactured and donated to the study by Karl Fazer Ab, Vantaa, Finland.

\section{Study design}

The study lasted 18 weeks altogether. During this period the subjects were instructed not to use antimicrobial medications, mouthwashes, or xylitol products, but to consume their normal, habitual diet, and continue their usual tooth brushing.

The study had a double-blind, crossover design as shown in Figure 1. The subjects were randomly allocated into two groups: half of the subjects used blue-coded gum first, and then the red-coded gum for four weeks, while the other half used the gums in reverse order. On the first appoint- 
ment the subjects were given instructions concerning the study. They were also given ordinary fluoridated toothpaste to be used during the study (free-of-charge) as well as the green-coded washout gum to be used during the wash-out periods. Since all subjects were gum users to start with it was considered that gum use also during the wash-out periods would ensure compliance. The subjects were told that all gums were sugar-free gum with benefits for dental health. Professional tooth-cleaning and dental examination were carried out for all participants $(\mathrm{AH})$.

Before the test periods the subjects received the test chewing gums. During the test periods, the subjects were instructed to use two chewing gum pieces at a time, three times a day, preferably after meals. The recommended chewing time was $5 \mathrm{~min}$. The gum consumption resulted in a daily polyol dose of approximately $6 \mathrm{~g}$. The recommendation for gum usage during the wash-out periods was less strict than for the test periods, i.e. 2-3 times a day. The subjects were instructed to refrain from tooth brushing and other oral hygiene procedures for 24 hours before sample collection and not to eat or drink for 1 hour before the sampling appointment. Compliance with the instructions provided was verified at the beginning of each appointment. At the appointments the subjects were also interviewed about amount and frequency of chewing gum use, and confounding factors like acute infectious diseases and use of antibiotics.
The subjects and researchers in Oulu were blinded throughout the study. Also the microbiological analyses in Turku were carried out blinded. The test code was revealed after the results had been fed into SPSS files. Thereafter one of the authors (ES) randomly chose the samples of five subjects from those subjects who harbored MS throughout the study to be sent to the US for DNADNA hybridization. The microbial analyses in the US were performed in a blinded fashion and the results were analyzed in Turku.

\section{Saliva/plaque sampling}

Paraffin-stimulated saliva was collected for 5 minutes. $100 \mu \mathrm{l}$ of the saliva was transferred to a transport tube containing $900 \mu \mathrm{L}$ Tryptic Soy Broth (Difco, MI, USA) with $10 \%$ glycerol v/v. The tube was stored at $-70^{\circ} \mathrm{C}$ before analyses. After the saliva collection dental floss attached to a floss-holder was used to collect plaque from "caries-prone" tooth sites, the mesial surfaces of two first molars $\left(D 16^{2}, D 36^{2}\right)$. The mid-parts of the flosses with the plaque samples were cut into a transport tube containing $1 \mathrm{ml}$ of transport medium. All available plaque was then collected with curettes, first from the left side of the mouth, and the plaque was suspended in EDTA buffer $10.25 \mathrm{M} \mathrm{NaOH}, 5 \mathrm{mM}$ Tris, $0.5 \mathrm{mM}$ EDTA), stored at $-70^{\circ} \mathrm{C}$, and mailed for microbiological analysis to Indiana University, Indianapolis, USA on dry ice. The plaque from the right side of the mouth was suspended in $1 \mathrm{ml}$ saline, and disperged by pumping the suspension back-

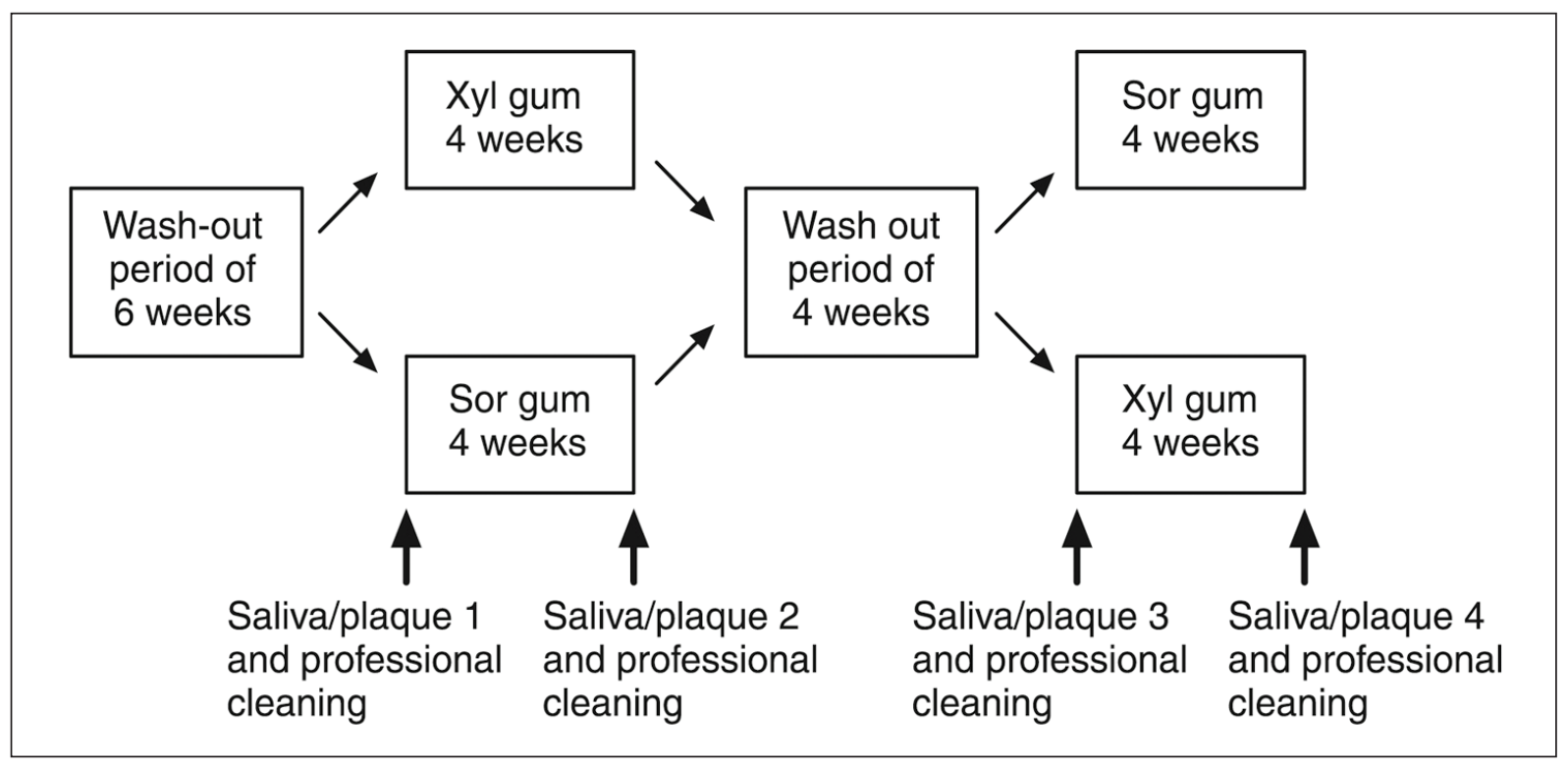

Figure 1. Study design. The 4-wk test periods when xylitol ( $\mathrm{Xyl}$ ) or the control, sorbitol (Sor), gums were used, were preceded by a 6-wk wash-out period. Between the test periods there was a 4 -wk washout period. Arrow indicates sampling appointments followed by a professional tooth cleaning. 
and-forth with a disposable pipette. Then, $500 \mu \mathrm{l}$ of the suspension was pipetted into a transport tube containing $2 \mathrm{ml}$ of transport medium and stored frozen at $-70^{\circ} \mathrm{C}$ until analysed. Except for the analyses performed in the US, all microbiological analyses were performed at the University of Turku, Turku, Finland, $620 \mathrm{~km}$ south of Oulu. The samples were mailed from Oulu to Turku frozen, on dry ice. The transport medium and the transport conditions have been tested earlier and they have shown excellent recoveries of streptococci and total facultatives. ${ }^{8}$

\section{Microbiological analyses}

For salivary analyses the transport tubes were thawed and vortexed for 1 minute. The plaque samples were further disperged with a mild 10 -second sonication at $4^{\circ} \mathrm{C}$. After 10 -fold serial dilutions the samples were plated on Mitis salivarius agars containing bacitracin (MSB; Difco, Detroit, MI, USA ${ }^{14}$ ), TYCSB agars (Difco ${ }^{15}$ ), Mitis salivarius agars (Difco), Rogosa agars (Difco) and blood agars (Orion Diagnostica). The MS grown on MSB agar were incubated for 2-3 days in a $7 \% \mathrm{CO}_{2}$ atmosphere, and the TYCSB plates anaerobically for 3 days at $37^{\circ} \mathrm{C}$. The identification of MS has been described in detail earlier. ${ }^{8}$ The numbers of MS were identified on the basis of colony morphology and counted by means of a stereomicroscope. The identification of $S$. mutans was based on con- sistent findings of "rough" colony morphology on the MSB plate, positive fermentation with sorbitol, mannitol, raffinose and melibiose, and negative dextran agglutination. Identification of $S$. sobrinus was based on "smooth" colonies on the MSB plate, positive fermentation with mannitol but negative with raffinose and melibiose, and positive dextran agglutination. The detection limit of the MS assay for saliva was $200 \mathrm{CFU} / \mathrm{ml}$ and for plaque 200 CFU/ sample. Total streptococci were grown for 2 days on Mitis salivarius agars at $37^{\circ} \mathrm{C}$. "Lactobacillilike" colonies were counted on the Rogosa agars and total facultatives on blood agars grown anaerobically for 2 days at $37^{\circ} \mathrm{C}$.

From those subjects who harbored MS throughout the study, five were randomly chosen for the DNA-DNA hybridizations ${ }^{16}$ performed at the Indiana University. Since the analyses are laborious and expensive to perform, it was decided that five samples would be analyzed first and then, depending on the results, the rest of the samples. Briefly, plaque DNA samples (Master Pure Gram Positive DNA Purification Kit, Epicentre Biotechnologies, Madison, WI, USA) were applied to a membrane using a vacuum slot blot, then UV crosslinked and hybridized to DIG-labelled species-specific probes using standard techniques (Roche Applied Sciences, Indianapolis, IN, USA) for hybridization and chemiluminescent detection. Specific probe sequences, $\operatorname{Tm}\left({ }^{\circ} \mathrm{C}\right)$ values and ref-

Table 1. Species - specific probes used for DNA hybridization.

\begin{tabular}{|c|c|c|c|c|}
\hline Microorganism & ATCC & Specific sequence & $\operatorname{Tm}\left({ }^{\circ} \mathrm{C}\right)$ & Ref. \\
\hline S. gordonii & 35105 & 5'-AGTTCCAAACTAGTCCATTG-3' & 56,3 & 17 \\
\hline S. sanguinis & 10556 & 5'-GACACACGGAATGCACTTG-3' & 60,2 & 18 \\
\hline S. mitis/oralis & 9811 & 5'-TGTTCCCAAGTGGAGCCAACCAAACT-3' & 66,2 & 19 \\
\hline S. parasanguis & 15912 & 5'-ACACTATAGTAGTGTTAAGC-3' & 54,2 & 18 \\
\hline S. salivarius & 13419 & 5'-CATATGTAATTACTTACATATAGATAGTAA-3' & 56,4 & 18 \\
\hline L. fermentum & 14932 & 5'-ATCAATCAATTGGGCCAAC-3' & 55,8 & 20 \\
\hline L. rhamnose & 7469 & 5'-CCTGCACACACGAAA-3' & 53,4 & 21 \\
\hline L. paracasei & 11582 & 5'-TGCCGCCGGCCAG-3' & 61,1 & 21 \\
\hline L. plantarum & 8014 & 5'-ACATTCTTCGAAACTTTGT-3' & 51,5 & 21 \\
\hline L. acidophilus & 4356 & 5'-TACCACTTTGCAGTCCTACA-3' & 58,4 & 21 \\
\hline A. viscosus & 43146 & 5'-ACGGAGGTCGGGAACGGTGGAAG-3' & 69,9 & 19 \\
\hline A. naeslundii & 19039 & 5'-CACTCATCCAGAACCAG-3' & 57,2 & 20 \\
\hline F. nucleatum & 25586 & 5'-CAACGCAATACAGAGTTGAGCCCTGCATT-3' & 67,4 & 19 \\
\hline V. parvula & 10790 & 5'-AATCCCCTCCTTCAGTGA-3' & 57,6 & 20 \\
\hline
\end{tabular}


erences are listed in Table 1. The following microorganisms were assessed: Actinomyces naeslundii, A. viscosus, Fusobacterium nucleatum, Lactobacillus acidophilus, L. fermentum, L. paracasei, L. rhamnose, L. plantarum, Streptococcus gordonii, S. oralis, S. parasanguis, S. salivarius, S. sanguinis, Veillonella parvula. The results were analyzed using Un-Scan-It software (Silk Scientific, Inc, Orem, UT, USA) and were expressed as pixels reflecting the number of microorganisms per sample.

\section{Statistical analysis}

The analysis of variance of repeated measures was used for longitudinal comparisons and for pair-wise comparisons the paired-samples $t$-test. Means were compared using independent samples $t$-test. The statistical package used was SPSS 14.0 for Windows. The level of statistical significance was set at $\mathrm{P}<.05$.

\section{RESULTS}

In the plaque samples collected from the "caries-prone" tooth surfaces a decrease in the MS counts was seen for all ten subjects in the xylitol gum group $(P<.01$; Figure 2$)$. No change was seen in the MS counts of the control/sorbitol gum group (Figure 2). Also the MS percentage of plaque decreased significantly in the xylitol gum group $(P<.01)$, while no change took place in the sorbitol gum group (Figure 2). Concerning salivary MS counts, no "xylitol effects" were detected.

The subjects were invited to participate in the study on the basis of a prescreening for the pres-

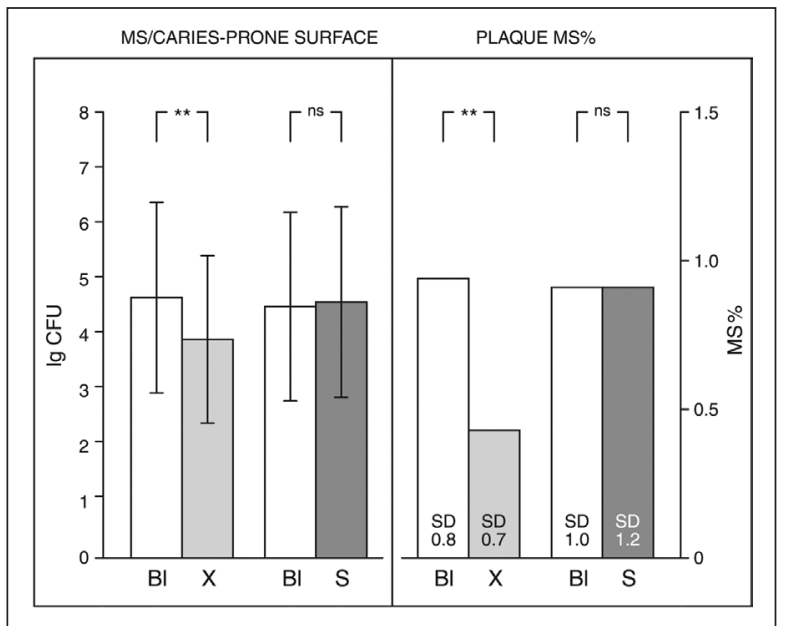

Figure 2. Counts of MS obtained from dental plaque present on caries-prone tooth surfaces, and plaque MS percentage figures calculated against total facultatives (mean $\pm S D ; n=10$ ). MS were cultured on TYCSB agar. Bl=baseline, $X=$ after use of $x y l i-$ tol gum, $\mathrm{S}=$ after use of sorbitol gum. ${ }^{* *}$ : $\mathrm{P}<.01$; ns=not significant. ence of MS. Two of the subjects showed rather low levels of MS at the prescreening and in the study proper they did not harbor detectable counts of MS in either plaque or stimulated saliva. Only one of the subjects harbored both $S$. mutans and S. sobrinus, with $S$. mutans being the dominant MS. MS culturing on MSB and TYCSB gave similar results: for some subjects the MS counts were higher on TYCSB, but in other respects the results were similar. In the counts of salivary total streptococci and lactobacilli no changes were seen during the study (Figure 3). The cross-over design did not affect the results. The two subgroups did not differ from each other when baseline counts of MS were compared before the two test periods. Also within the same subgroup the baseline values before the test periods did not differ.

All available plaque collected from the right side of the mouth was cultured for total facultatives at the University of Turku. There were no differences in the counts of total facultatives in plaque indicating that sampling was consistent and no changes in the amount of plaque took place during the study. The plaque from the left side of the mouth of five subjects was subjected to DNADNA hybridization at Indiana University. A. naeslundii, L. acidophilus, L. fermentum and S. parasanguis were detected in so few samples that they could not be included in the statistical analysis. L. rhamnose and L. plantarum were not detected in any of the samples. Only A. viscosus, $S$. gordonii, S. oralis and $S$. sanguinis were detected in the samples of all five subjects. The mean counts of the microor-

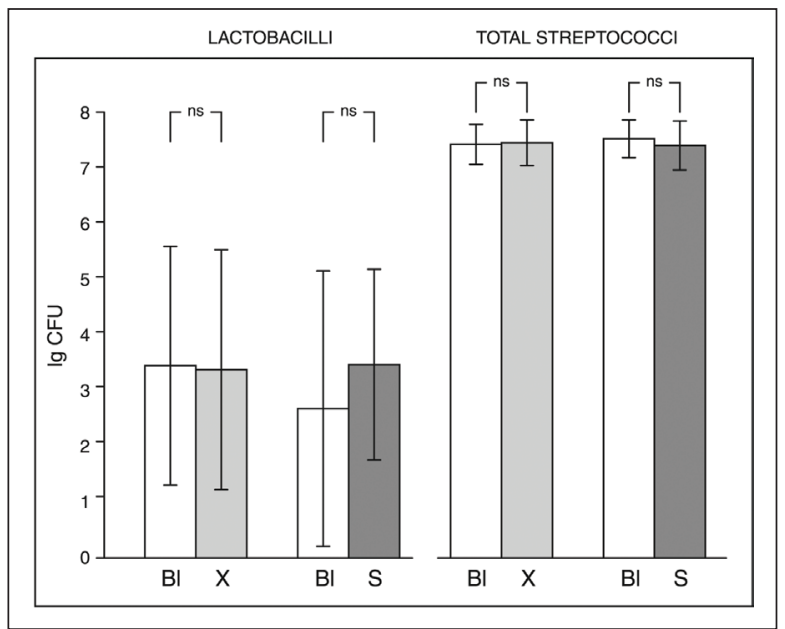

Figure 3. Salivary counts of lactobacilli and total streptococci (mean $\pm S D ; n=12)$. For further details, see Figure 2 . 
ganisms detectable in the samples of at least four subjects are shown in Table 2. No study-induced effects were detected in microbial counts of A. viscosus, F. nucleatum, L. paracasei, S. gordonii, S. oralis, S. salivarius, $S$. sanguinis or V. parvula. Since not even trends for study-induced effects on the flora could be observed in these analyses, the rest of the samples were omitted from the analyses.

\section{DISCUSSION}

The present short-term pilot study demonstrated that xylitol consumption did not affect the composition of oral flora except for a decrease in plaque MS. MS form only a small percentage of oral flora, thus it is surprising that so few studies have addressed this topic. Xylitol inhibits the growth of MS via the inducible fructose transport system. ${ }^{22}$ The degree of xylitol inhibition varies among MS strains. ${ }^{23-25}$ In addition to MS, S. gordonii possesses an inducible fructose pathway ${ }^{26}$ and could thus be inhibited by xylitol. Inhibition with xylitol is often addressed as the main mechanism by which xylitol consumption may reduce MS counts. ${ }^{7}$ Moreover, xylitol-induced changes in the virulence of MS have been proposed to contribute to this phenomenon. ${ }^{7}$ Recently, however, it was demonstrated in vitro that xylitol could decrease polysaccharide-mediated adhesion of MS contributing to plaque accumulation with a mechanism not dependent on growth inhibition. ${ }^{25}$

In vitro studies have suggested that MS are target organisms of xylitol. ${ }^{12,23}$ Loesche et al ${ }^{13}$ showed that consumption of xylitol chewing gum reduced MS in both plaque and saliva but not counts of $S$. sanguis. Studies have demonstrated growth inhibi- tion for $S$. mitis with xylito ${ }^{27}$ while $S$. milleri was not inhibited by it. ${ }^{28}$ Recently, it was shown that xylitol may inhibit in vitro both the growth and polysaccharide production of type strains and clinical isolates of MS as well as $S$. sanguinis and S. salivarius. ${ }^{25}$ In the present study, we found no effects induced by xylitol consumption on either total salivary streptococci counts or streptococci determined with the DNA-DNA hybridization technique, S. oralis, S. gordonii, S. salivarius or S. sanguinis. The present results support the idea that MS represent important target organisms of xylitol.

Several oral lactobacilli, L. acidophilus, L. paracasei, L. brevis and L. fermentum, also possess the fructose pathway and could thus be inhibited by xylitol. ${ }^{29-31}$ Loesche et al ${ }^{13}$ demonstrated a xylitolassociated decrease in MS counts, but no effects of lactobacilli. A recent clinical trial showed a xylitol-associated decrease in salivary lactobacilli. This result may, however, reflect a xylitol-induced elevation of the oral $\mathrm{pH}$ and thus an indirect effect on lactobacilli. ${ }^{32}$ Our results support the idea that xylitol consumption does not reduce counts of lactobacilli.

A. viscosus, F. nucleatum and V.parvula apparently do not possess the inducible fructose pathway but could, in theory, be affected for example by the changes xylitol consumption may have on oral $\mathrm{pH}$. All subjects harbored these organisms; but no trends to any study-induced changes could be detected in the counts of the microorganisms. Thus, xylitol appeared not to affect the levels of these microorganisms. In vitro studies support this idea; Actinomyces strains, $V$. dispar and F. nucleatum were not inhibited by xylitol. ${ }^{12,23}$

Table 2. The effect of chewing xylitol vs. sorbitol gum on the composition of plaque flora. The counts of each microorganism per plaque sample are expressed as pixels (mean \pm SD of logarithmic values). The pixels can be compared intraindividually within one subject but not interindividually. No statistically significant differences were found.

\begin{tabular}{lcccc}
\hline & \multicolumn{2}{c}{ Xylitol chewing gum } & \multicolumn{2}{c}{ Sorbitol chewing gum } \\
Microorganism & Baseline & After gum use & Baseline & After gum use \\
\hline A. viscosus & $4.2 \pm 0.7$ & $4.4 \pm 0.4$ & $4.4 \pm 0.4$ & $4.4 \pm 0.4$ \\
F. nucleatum & $1.4 \pm 3.1$ & $2.4 \pm 2.5$ & $1.2 \pm 3.0$ & $1.3 \pm 3.0$ \\
L. paracasei & $3.1 \pm 2.9$ & $3.1 \pm 2.8$ & $3.3 \pm 2.9$ & $3.1 \pm 2.9$ \\
S. gordonii & $4.1 \pm 0.5$ & $4.4 \pm 0.5$ & $4.5 \pm 0.4$ & $4.5 \pm 0.4$ \\
S. oralis & $3.7 \pm 0.4$ & $3.8 \pm 0.5$ & $2.7 \pm 2.7$ & $2.7 \pm 2.8$ \\
S. salivarius & $0.4 \pm 3.3$ & $1.5 \pm 3.2$ & $1.6 \pm 3.3$ & $2.8 \pm 2.7$ \\
S. sanguinis & $4.2 \pm 0.4$ & $4.3 \pm 0.4$ & $4.3 \pm 0.4$ & $4.1 \pm 0.7$ \\
V. parvula & $3.6 \pm 0.6$ & $4.0 \pm 0.4$ & $4.2 \pm 0.3$ & $2.9 \pm 2.7$ \\
\hline
\end{tabular}


Rather poor "xylitol effects" on MS were demonstrated in the present study compared to earlier studies employing similar xylitol doses and consumption frequencies. ${ }^{3,33}$ The study subjects were highly motivated dental/medical students. The fact that significant decreases were seen in the MS of the plaque samples from the caries-prone areas and plaque MS percentages indicates good compliance. Ten subjects were daily xylitol consumers and two used xylitol approximately twice a week before the study started. In spite of the long washout period before the study and the professional tooth cleaning, habitual xylitol consumption may cause long-term changes in the MS counts as discussed recently. ${ }^{34}$ In this study, the cross-over design was not the reason for the poor "xylitol effects"; the confounding factor was most probably the fact that the subjects were xylitol consumers to start with. Long-term consumption of xylitol has been connected with the selection of naturally occurring, so-called "xylitol-resistant" MS, not inhibited by xylitol. ${ }^{35}$ This phenomenon may at least partly explain why the "xylitol effects" were relatively poor in this study. The "resistancy" phenomenon has, however, never been demonstrated for any other oral microorganisms than MS. Thus, within the limitations caused by the small number of subjects, our results concerning the composition of the oral flora in general should be valid.

\section{CONCLUSIONS}

Within the limitations of this pilot study, xylitol consumption reduced MS counts but appeared not to affect the microbial composition of plaque or saliva in general.

\section{ACKNOWLEDGEMENTS}

The excellent technical assistance provided by biomedical research technician Oona Hällfors is gratefully acknowledged.

\section{REFERENCES}

1. Havenaar R, Huis in 't Veld JHJ, Backer Dirks O, de Stoppelaar JD. Some bacteriological aspects of sugar substitutes. Health and Sugar Substitutes. Proc. ERGOB Conf., Geneva 1978, pp. 192-198 (Karger, Basel 1978).
2. Mäkinen KK, Mäkinen PL, Pape HR Jr, Peldyak J, Hujoel P, Isotupa KP, Söderling E, Isokangas PJ, Allen P, Bennett C. Conclusion and review of the Michigan Xylitol Programme (1986-1995) for the prevention of dental caries. Int Dent J $1996 ; 46: 22-34$

3. Ly KA, Milgrom P, Rothen M. The potential of dental-protective chewing gum in oral health interventions. J Am Dent Assoc 2008;139:553-563.

4. Machiulskiene V, Nyvad B, Baelum V. Caries preventive effect of sugar-substituted chewing gum. Community Dent Oral Epidemiol 2001;29:278-288.

5. Hayes C. Xylitol gum decreases the decayed, missing, and filled surfaces (DMFS) score by an average of 1.9. J Evidence-Based Dent Pract 2002;2:14-15.

6. Milgrom P, Ly KA, Tut OK, Mancl L, Roberts MC, Briand K, Gancio MJ. Xylitol pediatric topical oral syrup to prevent dental caries: a double-blind randomized clinical trial on efficacy. Arch Pediatr Adolesc Med 2009;163:601-607.

7. Söderling EM. Xylitol, mutans streptococci, and dental plaque. Adv Dent Res 2009;21:74-78.

8. Söderling $E$, Isokangas $P$, Pienihäkkinen $K$, Tenovuo J. Influence of maternal xylitol consumption on acquisition of mutans streptococci by infants. J Dent Res 2000;79:882887.

9. Thorild I, Lindau B, Twetman S. Effect of maternal use of chewing gums containing xylitol, chlorhexidine or fluoride on mutans streptococci colonization in the mothers' infant children. Oral Health Prev Dent 2003;1:53-57.

10. Fontana M, Catt D, Eckert GJ, Ofner S, Toro M, Gregory $\mathrm{RL}$, Zandona AF, Eggertsson H, Jackson R, Chin J, Zero D, Sissons $\mathrm{CH}$. Xylitol: effects on the acquisition of cariogenic species in infants. Pediatr Dent 2009;31:257-266.

11. Nakai Y, Shinga-Ishihara C, Kaji M, Moriya K, MurakamiYamanaka K, Takimura M. Xylitol gum and maternal transmission of mutans streptococci. J Dent Res 2010;89:56-60.

12. Bradshaw DJ, Marsh PD. Effect of sugar alcohols on the composition and metabolism of a mixed culture of oral bacteria grown in a chemostat. Caries Res 1994;28:251-256.

13. Loesche WJ, Grossman NS, Earnest R, Corpron R. The effect of chewing xylitol gum on the plaque and saliva levels of Streptococcus mutans. J Am Dent Assoc 1984;108:587592.

14. Gold OG, Jordan HV, Van Houte J. A selective medium for Streptococcus mutans. Arch Oral Biol 1973;18:1357-1364.

15. Van Palenstein Helderman WH, Isseldijk $M$, Huis in't Veld $\mathrm{JH}$. A selective medium for the two major subgroups of the bacterium Streptococcus mutans isolated from human dental plaque and saliva. Arch Oral Biol 1983;28:599-603. 
16. Gellen LS, Wall-Manning GM, Sissons CH. Checkerboard DNA-DNA hybridization technology using digoxigenin detection. Methods Mol Biol 2007;353:39-67.

17. Al-Ahmad A, Auschill TM, Braun G, Hellwig E, Arweiler NB. Overestimation of Streptococcus mutans prevalence by nested PCR detection of the 16S rRNA gene. J Med Microbiol 2006;55:109-113.

18. Chen CC, Teng LJ, Kaiung S, Chang TC. Identification of clinically relevant viridans streptococci by an oligonucleotide array. J Clin Microbiol 2005;43:1515-1521.

19. Suzuki N, Yoshida A, Nakano Y. Quantitative analysis of multi-species oral biofilms by TaqMan Real-Time PCR. Clin Med Res 2005;3:176-185.

20. Becker MR, Paster BJ, Leys EJ, Moeschberger ML, Kenyon SG, Galvin JL, Boches SK, Dewhirst FE, Griffen AL. Molecular analysis of bacterial species associated with childhood caries. J Clin Microbiol 2002;40:1001-1009.

21. Haarman M, Knol J. Quantitative real-time PCR analysis of fecal Lactobacillus species in infants receiving a prebiotic infant formula. Appl Environ Microbiol 2006;72:2359-2365.

22. Trahan L, Bareil M, Gauthier L, Vadeboncoeur C. Transport and phosphorylation of xylitol by a fructose phosphotransferase system in Streptococcus mutans. Caries Res 1985;19:53-63.

23. Vadeboncoeur C, Trahan L, Mouton C, Mayrand D. Effect of xylitol on the growth and glycolysis of acidogenic oral bacteria. J Dent Res 1983;62:882-884.

24. Miyasawa-Hori H, Aizawa S, Takahashi N. Difference in the xylitol sensitivity of acid production among Streptococcus mutans strains and the biochemical mechanism. Oral Microbiol Immunol 2006;21:201-205.

25. Söderling EM, Hietala-Lenkkeri AM. Xylitol and erythritol decrease adherence of polysaccharide-producing oral streptococci. Curr Microbiol 2010;60:25-29.

26. Loo CY, Mitrakul K, Voss IB, Hughes CV, Ganeshkumar N. Involvement of an inducible fructose phosphotransferase operon in Streptococcus gordonii biofilm formation. $J$ Bacteriol 2003; 185:6241-6254

27. Kontiokari T, Uhari M, Koskela M. Effect on xylitol on growth of nasopharyngeal bacteria in vitro. Antimicrob Agents Chemother 1995;39:1820-1823.

28. Rogers AH, Pilowsky KA, Zilm PS, Gully NJ. Effects of pulsing with xylitol on mixed continuous cultures of oral streptococci. Aust Dent J 1991;36:231-235.

29. Saier MH Jr, Ye JJ, Klinke S, Nino E. Identification of an anaerobically induced phosphoenolpyruvate-dependent fructose-specific phosphotransferase system and evidence for the Embden-Meyerhof glycolytic pathway in the heterofermentative bacterium Lactobacillus brevis. J Bacteriol 1996;178:314-316.
30. Kaplan H, Hutkins RW. Fermentation of fructooligosaccharides by lactic acid bacteria and bifidobacteria. Appl Environ Microbiol 2000;66:2682-2684.

31. Helanto M, Aarnikunnas J, Palva A, Leisola M, Nyyssöla A. Characterization of genes involved in fructose utilization by Lactobacillus fermentum. Arch Microbiol 2006;186:51-59.

32. Mäkinen KK, Alanen $P$, Isokangas $P$, Isotupa $K$, Söderling E, Mäkinen PL, Wenhui W, Weijian W, Xiaochi C, Yi W, Boxue Z. Thirty-nine-month xylitol chewing-gum programme in initially 8-year-old school children: a feasibility study focusing on mutans streptococci and lactobacilli. Int Dent J 2008;58:41-50.

33. Milgrom P, Ly KA, Roberts MC, Rothen M, Mueller G, Yamaguchi DK. Mutans streptococci dose response to xylitol chewing gum. J Dent Res 2006;85:177-181.

34. Mäkinen KK. An end to crossover designs for studies on the effect of sugar substitutes on caries? Caries Res 2009;43:331-333.

35. Trahan L. Xylitol: a review of its action on mutans streptococci and dental plaque - its clinical significance. Int Dent $J$ 1995;45:77-92. 